\title{
Arte, salud mental y trabajo social
}

\author{
Art, mental health \\ and social work
}

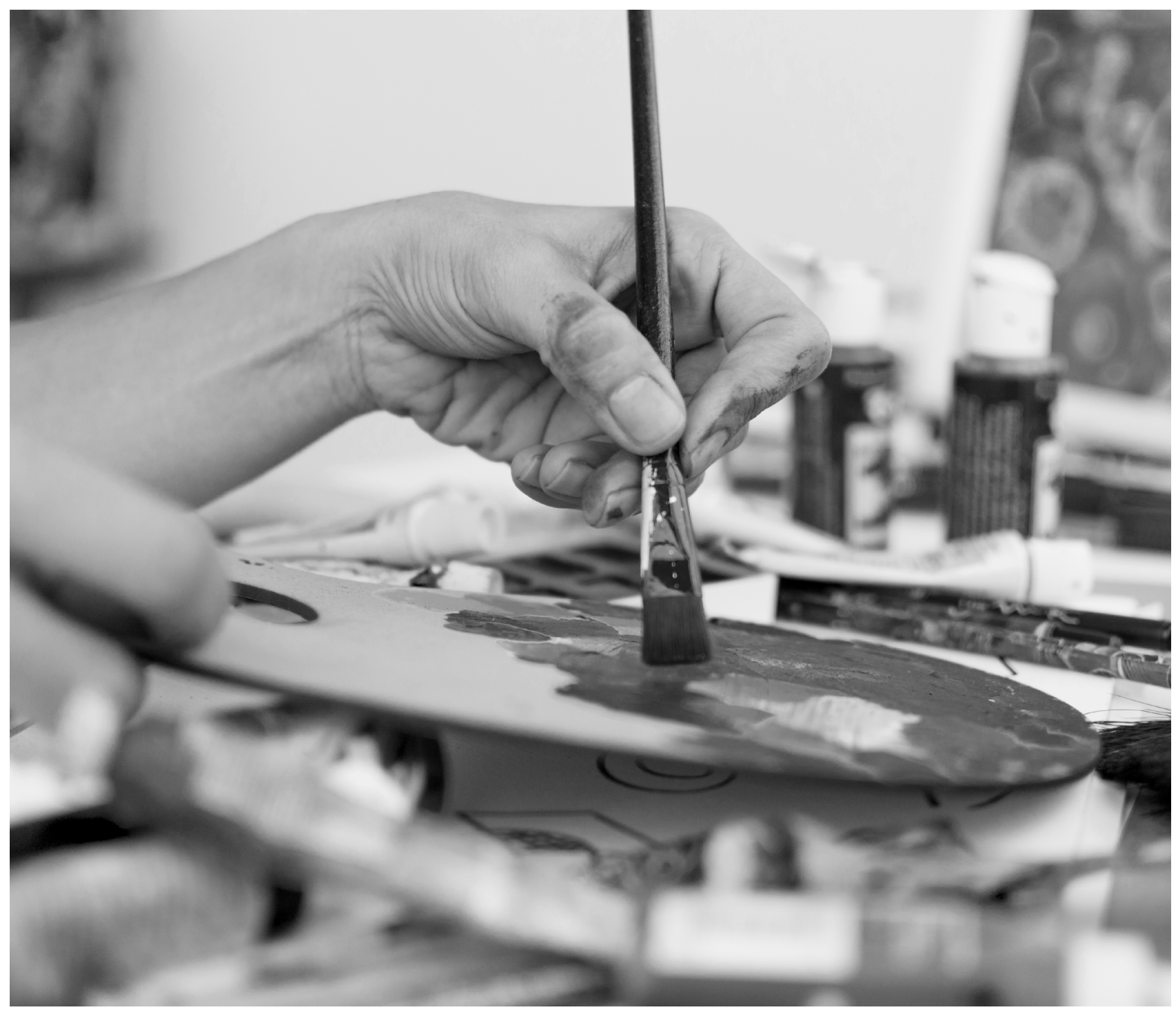




\title{
Arte, salud mental y trabajo social' Art, mental health and social work
}

\author{
Betina Mariel-Bovino²
}

Artículo recibido en marzo de 2018; artículo aceptado en septiembre de 2018.

Este artículo puede compartirse bajo la Licencia Creative Commons Atribución-NoComercial-Compartirlgual 4.0 Internacional

y se referencia usando el siguiente formato: Mariel-Bovino, B. (2019). Arte, salud mental y trabajo social.

I+D Revista de Investigaciones, 13 (1), 45-55. DOI: https://doi.org/10.33304/revinv.v13n1-2019004

\section{Resumen}

Este artículo aborda la construcción, en el campo de la salud mental, de las estrategias de intervención profesional del Trabajo Social en relación con el Arte en la Provincia de Santa Fe, Argentina. Para ello, se propuso identificar las estrategias de intervención del trabajo social en salud mental, y describir las articulaciones y construcciones de dichas estrategias con las diferentes disciplinas que intervienen en dicho campo. Asimismo, se intenta determinar los aportes del arte en la intervención con sujetos con padecimiento subjetivo. Se determinó así que el arte puede operar como mecanismo preventivo del desgaste profesional y de las posibles repercusiones que este genera en los individuos.

Palabras clave: trabajo social, estrategias de intervención, arte, salud mental.

\begin{abstract}
This article deals with the construction in the field of mental health of the strategies of the professional intervention of social work in relation to art, in the Province of Santa Fe, Argentina. To do this, it was proposed to identify intervention strategies of social work in mental health; describe the articulations and constructions of said strategies with the different disciplines that intervene in that field; likewise, we try to determine the contributions that art makes in the intervention with subjects with subjective suffering. Determining that art can operate as a preventive mechanism of professional burnout and the possible repercussions that it generates on individuals.
\end{abstract}

Keywords: social work, intervention strategies, art, mental health.

\section{Introducción}

"El arte es una respuesta a la vida. Ser artista es emprender una manera riesgosa de vivir, es adoptar una de las mayores formas de libertad" - Antonio Berni (UNESCO, 2016).

La Federación Internacional de Trabajo Social (FITS) define al Trabajo Social como la profesión que promueve el cambio social, la solución de problemas en las relaciones humanas, el fortalecimiento y la liberación de las personas para incrementar el bienestar. Los principios de los Derechos Humanos y la justicia social son fundamentales para el trabajo social. Esta definición aporta, sin dudas, cierta especificidad a la intervención del trabajador social

1. Artículo de revisión. Enfoque cualitativo, resultado de un proyecto de investigación en curso, perteneciente al Doctorado en Trabajo Social. Facultad de Ciencia Política y Relaciones Internacionales. Universidad Nacional de Rosario (Rosario, Argentina). Dirección Riobamba 65 bis.

2. Licenciada en Trabajo Social. Universidad Nacional de Rosario (Rosario, Argentina). Doctoranda en Trabajo Social. Docente investigadora de la ciudad de Rosario (Argentina). Dirección: Riobamba 600 bis. Integrante del Centro de Investigación en Campos de Intervención del Trabajo Social (Ciecits) Facultad Ciencia Política y Relaciones Internacionales (RRII). ORCID ID: https://orcid.org/0000-0002-8597-7843 Correo electrónico personal: ciecitsunr@gmail.com. Correo electrónico institucional: betibovino@hotmail.com. 
en el ámbito de la Salud Mental, entendida esta como un proceso determinado por componentes históricos, socioeconómicos, culturales, biológicos y psicológicos, cuya preservación y mejoramiento implica una dinámica de construcción social vinculada a la concreción de los derechos humanos y sociales de toda persona - Art 3. Ley 26.657 - (Ministerio de Salud, 2013). Preguntarse sobre la intervención del trabajador social en Salud Mental pareciera ser un debate superado y analizado en demasía. Pero los avatares sociales e históricos por los que atraviesa una comunidad, el discurso imperante y los conflictos que como sociedad nos afectan, demandan formas más específicas y más creativas de intervención profesional.

Las problemáticas que se abordan en el campo de la Salud Mental se presentan como multicausales, determinadas por diversos factores. El conocimiento de la situación, así como la fundamentación teórica, permiten analizar y definir la estrategia de intervención.

Los profesionales del trabajo social de un equipo de salud frecuentemente identifican que las personas con algún padecimiento mental con quienes trabajan realizan algunas prácticas, y presentan conductas o comportamientos que para otros compañeros del mismo equipo serían impensables o poco probables. Es común que apreciaciones diagnósticas de internación de por vida, agotamiento de la capacidad de autonomía y de la de vivir solo, pérdida de interés en actividades de la vida cotidiana, imposibilidad de desarrollar una vida en común con otras personas y otras apreciaciones diagnósticas, sean desmentidas por la realidad de los usuarios una vez que se les permite y acompaña el proceso de autonomía, cuando se permiten ver "cómo se producen los efectos de verdad en el interior de los discursos que no son en sí mismos ni verdaderos ni falsos" (Foucault, 1998).

Uno de los principales obstáculos que enfrenta la profesión del Trabajo Social y su formación académica, es la ausencia de materiales escritos que den cuenta de la especificidad y aplicación de las técnicas de actuación profesional. Durante años, se ha estudiado la importancia, las características y las modalidades de aplicación de las técnicas en otros campos disciplinares, pero el porqué y el para qué de las mismas en esta disciplina, se ha naturalizado o silenciado asumiéndose como obvio (Tonon, 2005).

Los trabajadores sociales están cotidianamente vinculados con las personas con padecimiento mental precisamente en este ámbito de la experiencia donde se dialoga y se discute sobre la experiencia que ellos hacen y tienen de su propia existencia. Esto nos pone dentro de la discusión sobre las capacidades que poseen las personas con padecimiento mental, o las capacidades que tienen, aún con padecimiento mental. Este lugar es donde la persona trata de afrontar la realidad de significar al mundo, su presente y sus obras. Donde, más allá de tomar esta realidad desde una forma limitada o incompleta, es la forma que esta persona utiliza para comunicarse con el mundo y para comunicar al mundo lo que es, proyecta y hace en tanto persona. Es allí donde la Salud Mental, el Arte y la intervención profesional se encuentran.

\section{Intervenciones sin fronteras}

¿Por qué nos interesa el arte? Porque es el proceso que permite a los individuos cruzar sus fronteras, sobrepasar las limitaciones, llenar sus vacíos y colmarse a sí mismos. El arte no es una condición, es un proceso en el que lo oscuro dentro de las personas se vuelve transparente (Grotowski, 1992).

Kandinski (1989) afirma que el arte, capaz de evolucionar, se basa también en su época espiritual, pero no solo es eco y espejo de esta, sino que contiene una energía profética vivificadora que actúa y amplia profundamente. La vida espiritual, en la que también se halla el arte, considerado este como uno de sus más fuertes agentes, es un movimiento complejo pero determinado, traducible a términos simples, que conduce hacia adelante y hacia arriba. El color suscita dos tipos de efectos: uno externo, material que produce sensaciones puramente físicas que duran poco y cuyo efecto prácticamente desaparece al cesar el estímulo; y uno interno, un efecto psíquico donde el color adquiere un sentido interior y hace que el alma se emocione. Se siente, se piensa, se vive: una triada indiscernible de creación abierta al devenir.

Siguiendo a Deleuze \& Bacon (2002), el arte es una cuestión de videncia, eso que desborda y estalla toda percepción conocida para liberar un universo intangible de sinsentido. El arte es vidente porque invoca las sensaciones que persisten y encarnan el acontecimiento para abrir en lo finito toda la potencia del infinito. Es una apertura que desprende y hace vibrar las sensaciones, las acopla y las abre, en un movimiento de composición y conservación de su infinitud; y el artista es creador de composiciones abiertas que inventan nuevos afectos, los muestra en su obra y los hace devenir en el mundo como línea trazada en el universo. El arte es considerado como una forma de constituir nuevos modos de vida, donde el pensamiento toma fuerza inactual e introduce una apertura sensible jamás percibida, donde pensar deviene una experiencia vital, y sentir es una variación del pensamiento. Por tanto, el arte como modo creativo de pensamiento es el poder que afirma la vida, el que abre las zonas de lo indiscernible, ya que en sus distintas manifestaciones funciona como elemento de subjetivación en las personas 
con padecimientos mentales; es transmisor de mensajes hacia el exterior e interior del sujeto; asimismo, interpela y promueve la participación. Devenir la vida en arte es un verdadero vehículo de integración y reinserción social. Arte y creatividad son instrumentos válidos en el abordaje de las problemáticas en Salud Mental, porque "el ser humano puede verse en el acto de ver, obrar, sentir y pensar. Puede sentirse sintiendo, verse viendo y puede pensarse pensando" (Boal, 2004). El interés del arte consiste en liberar espacios de vida para componer una topología dinámica de las multiplicidades.

Al definir estrategias de intervención para el tratamiento, externación y seguimiento, nos proponemos también desarrollar capacidades, de tal manera que los sujetos con padecimiento subjetivo puedan hacer frente a sus problemas actuales y a potenciales situaciones conflictivas, redescubriendo sus capacidades y recursos personales a partir del arte, interroga las prácticas de los profesionales de Trabajo Social.

Las estrategias de intervención de los trabajadores sociales implican crear dispositivos en la comunidad, en el medio natural del sujeto; $y$ es por medio del arte que se genera una implicación política en lo institucional, lo social y lo cultural. El arte les da voz como sujetos de derechos. Participar tiene efectos importantes en la subjetividad lastimada. Hay problemas concretos como la carencia de vivienda, la falta de empleo, la precariedad en las relaciones sociales, y es allí donde las estrategias de intervención profesional posibilitan el acompañamiento a las personas para poder volver a empoderarse e identificarse como una persona capaz, activa, parte de un colectivo.

El arte es considerado no tanto como una discusión sobre un objeto particular vinculado tradicionalmente al orden estético, bello, sublime, etc., sino como un problema del pensamiento mismo. Específicamente, el arte es un problema del pensamiento en directa relación con la vida, donde interesan más los efectos materiales que introducen nuevos horizontes vitales que las figuras posibles de una obra novedosa (Mengue, 2008). En este sentido, el arte es un modo particular de captura de fuerzas infinitas y la obra de arte una producción de signos que efectúan la génesis sensible del pensamiento. Así, el arte se presenta, en la filosofía deleuziana, como una expresión creativa del pensamiento, en tanto que es un modo de componer el caos, desde el trazado de un plano de composición en el que se instauran bloques de sensaciones que perduran por efecto de ciertas figuras estéticas.

Se ha analizado la "locura" en su dimensión histórica a partir de diferentes perspectivas. Autores como Goffman (1961, 1997) y Foucault (1961) se han pronunciado críticamente desde las Ciencias Sociales en relación con las rígidas estructuras y la carencia de derechos. Desde el disciplinamiento: la construcción social de la locura y la medicalización del control social, el impacto del encierro y las prácticas terapéuticas en la subjetividad del llamado "loco", las relaciones de poder instituidas en el manicomio que evidencian las manipulaciones operadas por los portadores de un saber legitimado socio-históricamente.

Un estudio realizado por Murekian (2002) en relación con la salud mental en el proceso de desmanicomialización y como eje de interpretación para la aplicación de políticas públicas, realizado en la ciudad de Viedma, Argentina, da cuenta de que la memoria colectiva sujeta a las representaciones hegemónicas de corte manicomial comenzó a dar paso a nuevos modos de representación, ampliando y diversificando los recursos simbólicos frente al contacto cotidiano y público con la locura, aun con todas las resistencias consecuentes.

Desde la Medicina, autores como Kraepelin (2008) sostienen que el surgimiento de la Psiquiatría como guardadora del individuo con padecimiento mental asume el rol disciplinador, permeando las representaciones sociales acerca de la locura que ha construido la sociedad.

Galende (1997) ha desarrollado en Argentina la relación entre lo social, la locura y sus efectos en la sociedad.

En el Trabajo Social, y en los orígenes de la profesión Richmond (1917), introduce una intencionalidad interventiva que supone una relación dinámica entre conocer-intervenir-transformar integrando lo individual y lo colectivo, tomando en cuenta las relaciones sociales y el ambiente en el que está inmerso el sujeto.

En Argentina, desde la intervención profesional de Kisnerman (1984) y Carballeda (2008), se realizan importantes análisis de las políticas sociales estatales e instituciones en salud mental, centrados en las reformas y contrarreformas de las políticas de salud mental. También Alberdi (2012) analiza exhaustivamente el tema en Argentina. No dejan de estar presentes en esta línea Rozas (2001), con su profundo análisis de la cuestión social, y otros colegas como Barg (2006), en relación con el abordaje interdisciplinario de la salud mental con un enfoque centrado en la familia. Amico (2005) se centra en la intervención del Trabajo Social en dispositivos alternativos de acción y allí articula locura con la noción de imaginario social, el sentido vigente de la institucionalización y la noción entre necesidades y derechos en las nuevas formas del padecimiento subjetivo.

En cuanto al análisis específico de las relaciones entre 
el hospital psiquiátrico y la familia de los pacientes, se encuentra el trabajo de Birman y Serra (1988) en Brasil. Por otro lado, Amarante et al. (2013) proponen, en el marco del movimiento brasileño de desinstitucionalización psiquiátrica, visualizar un "campo artístico-cultural" separado del "campo de la atención psicosocial", con la intención de correr el eje del discurso psíquico/ psiquiátrico hacia una racionalidad estética que permita formas de subjetivación alternativas.

Siguiendo esta idea, Guattariy Rolink (2006) consideran que la actividad artística no es solo el acto concreto de creación, sino también todo lo que se da en torno a eso, incluyendo lo inasible, aquello que no se puede poner en palabras, algo que irrumpe, que no es planificado ni esperado. Esta producción artística permite no solo experiencias estéticas materiales e inmateriales: obras, sino que también efectos sobre el cuerpo, nuevas subjetividades.

Para Carballeda (2007), el arte desde su vinculación con el proceso creativo

Se presenta como la posibilidad de construir y elaborar nuevas respuestas ante situaciones, desde el sujeto, el grupo o la comunidad. La creatividad en sí misma, como dispositivo de intervención, implica el redescubrimiento de la importancia de lo grupal, la relación entre lo grupal y lo comunitario, la promoción de nuevas formas de comunicación, y, por último, la posibilidad de explorar posibilidades expresivas con el cuerpo en relación con el espacio. Lo creativo como estrategia de intervención lleva, en otro plano, a crear, sustentar o reconstruir la identidad.

Analizando el planteamiento de Cazzaniga (2006), preguntarse acerca del "otro" indefectiblemente es la pregunta por la que los profesionales en el tema trabajan. La autora considera firmemente que si el "otro", inespecífico, no interpela, no desordena, es imposible que pueda construirse una relación dialógica; si no se vive la presencia del otro como pregunta, no se podrán ensayar múltiples y colectivas respuestas, se vaciará de sentido y contenido la tan mentada dimensión ético-política de la profesión y también de las alternativas que componen la caja de herramientas del trabajador social. Tonon (2005), considera que el arte es una herramienta potenciadora de la expresión e identificación, el alcance y proyección del instrumental depende de la postura crítica y de la creatividad de los sujetos profesionales e institucionales que lo implementan.

Retomando las ideas deVelez (2003), surge la necesidad de una mirada epistemológica, metodológica, ética y política que posibilitan leer, comprender y analizar los sujetos, los contextos y la situación social donde el Trabajador Social actúa. Esto implica abordar la intervención en términos estratégicos ya que, de acuerdo con Gónzalez (2007), el Trabajo Social es una profesión centrada en la intervención de lo social, con el objetivo de modificar situaciones particulares que aborda en su ejercicio cotidiano.

El Trabajo Social surge y se desarrolla simultáneamente con la presencia del manicomio en América Latina. Desde su surgimiento la intervención del Trabajo Social en el campo de la salud mental se tornó una especialización particular de la profesión (Amico, 2005). Este posicionamiento demandó para el desarrollo profesional el uso de saberes y métodos asociados a la psicología, la sociología y la psiquiatría. La intervención se desplazaba hacia el problema individual, ajeno a las condiciones estructurales del propio capitalismo y por lo tanto ubicando esta intervención en el terreno de lo patológico como disfuncionalidades a ser corregidas. Teniendo en cuenta los aspectos sociales dentro de las instituciones psiquiátricas, podría decirse que el Trabajo Social desde su fundación intervino en general desde una perspectiva centrada en la disciplina y la normalización del "otro desviado" para integrarlo al sistema. El servicio social surge en el escenario histórico con una identidad atribuida que expresaba una síntesis de las prácticas sociales precapitalistas, represoras y controladoras en pos de la definitiva consolidación del sistema capitalista.

En la actualidad, el Trabajo Social se enfrenta a nuevos desafíos que implican continuidades y rupturas con respecto a la práctica profesional tradicional y exigen, además de grandes destrezas técnicas, el desarrollo de sólidas bases teóricas que permitan convertir el oficio del profesional en una actuación dotada de sentido y orientada según este mismo.

Pensar la intervención en términos estratégicos implica no subsumirla a una racionalidad univoca y excluyente, sino justificarla y sostenerla en una perspectiva teóricometodológica fundada, considerando los intercambios y reciprocidades en la vida cotidiana, desde lo subjetivo, lo histórico, lo cultural, lo identitario, lo singular, lo comunitario y también lo simbólico.

Deleuze (1996) sostiene que:

No se escribe con las neurosis. Las neurosis, las psicosis, no son pasadizos de vida, sino estados en los que se cae cuando el proceso se interrumpe, se inmoviliza, se cierra. La enfermedad no es proceso, sino detención del proceso. Así, el escritor cuando escribe no lo hace como enfermo, sino como curador de sí y del mundo. El mundo es el conjunto de síntomas cuya enfermedad se confunde con el hombre. La literatura se presenta, entonces, como 
acción que sana: no es que el escritor posea una gran salud, sino que tiene una pequeña, pero irresistible salud, que proviene de las cosas demasiado grandes, fuertes e irrespirables que ha visto y escuchado. A partir de esto, el escritor regresa con los ojos enrojecidos y los tímpanos perforados. La salud como literatura, [como arte], consiste en inventar poblaciones que faltan.

Es la construcción colectiva la que nos devuelve un modo de estar diferente en donde la expresión adviene. La continuidad con la tarea hace que los cuerpos vayan modificándose en su estar y su transitar, por el taller, por el hospital, en la vida cotidiana. Cuerpos institucionalizados, desganados, encorvados, llenos de pastillas, dormidos, que cambian su postura, que sonríen, que se vuelven a habitar. Se trata, entonces, de inventar escenarios que den espacio y tiempo para que la diversidad de potencialidades pueda expresarse y combinarse en nuevos posibles; para que el no-lugar, aquel donde no se encuentran simbolizadas ni la identidad, ni la interacción, ni la historia (Auge, 2000) se conviertan en resistencia para componer con los fragmentos del manicomio otro orden de relación: deconstruir para inventar otras formas.

En Argentina, el regreso a la democracia en 1983 permitió la emergencia de múltiples prácticas y discursos que favorecieron el establecimiento de espacios de transformación junto a la elaboración de políticas desmanicomializadoras. Se intenta una experiencia en Río Negro, donde el Hospital Psiquiátrico de Allen se transforma en Hospital General, con un servicio de salud mental que logra cierta legitimación a través de la Ley 2440, promulgada en 1991. En Santa Fe se desarrolla un proceso similary también en Córdoba, aunque con logros diferentes.

En Buenos Aires, en el Hospital Borda, se convocó a distintos profesionales, entre ellos a los doctores José Grandinetti, Ricardo Grimnson y Alberto Méndez, para trabajar en el tema de salud mental. Aunque la experiencia no se concretó, quedaron instaladas ciertas propuestas que cuestionaron el modelo de internación y de tratamientos vigentes; entre ellas, la del Frente de Artistas del Borda. Este grupo se establece a fines del año 1984, con el desarrollo de talleres de los que participan internos, pacientes que se atienden en los consultorios ambulatorios y externados.

La experiencia de la internación durante períodos prolongados en grandes hospitales psiquiátricos, además de generar una serie de efectos negativos como la sobremedicación, la iatrogenia, el aislamiento, la ruptura de lazos sociales y la pérdida de la dimensión singular que adquiere el sufrimiento psíquico para quien lo padece, tiene como resultado una importante pérdida de la sensibilidad, delpensamientocríticoydelacapacidadcreativa, generando aquello que Goffman (1974) denomina "mutilación del yo", esto es, la continua mutilación de la singularidad del sujeto, que tiene su origen en la homogeneización subyacente a los mecanismos disciplinares, ya sea por la sumisión de quien se encuentra internado a diversos procedimientos que deterioran su identificación con antiguos roles sociales o por su transformación en mero objeto.

Para Clark (1998) el "estado de arte" es un estado de creación, que corre a través de todas las dimensiones de la vida, incluyendo la vida cotidiana. El propósito del estado de arte es hacer de la vida una obra de arte, crear desde un estado subjetivo en el que se puede hacer frente a la temporalidad de las formas y cambiar las formas de la experiencia. Generar la libertad de ser diferentes, vivir la experiencia de la singularidad. Por lo tanto, no solo se trata de la construcción de un producto artístico, socialmente aceptado y valorado, sino también la creación de la existencia objetiva y subjetiva. Metafóricamente hablando, siguiendo a esta autora, se puede decir que abandonar la actitud pasiva de "paciente" y llenar el escenario del teatro o la pista de baile es también dejar la posición subjetiva del espectador y ocupar el papel protagónico en la propia vida.

La reinvención del arte es condición necesaria para que esta pueda intervenir en la transformación del hombre y del mundo, superando las categorías del arte, tomándolas como categorías de vida, sea por la estética de lo cotidiano, sea por la recreación del arte como vida que en salud mental es condición indispensable para la superación de un modelo médico-hegemónico, hospital o céntrico, excluyente.

Generalmente, el "manicomio" produce un efecto en las personas con muchos años de internación, por el cual va dinamitando las capacidades que tiene un ser humano de pensar, sentir y hacer. Va destruyendo, estallando las pasiones, los deseos, los vínculos personales dentro y fuera del hospital; va convirtiendo progresivamente al sujeto en un objeto. Es precisamente en este punto donde radica la importancia del arte, dado que es el encargado de recuperar el deseo, la pasión, la actitud; despierta aptitudes, comienza a producir vínculos grupales, la persona comienza a pensar, a sentir, y a ocuparse de sí mismo, de su cuerpo, empieza a proyectar, puede cantar, pintar, actuar, escribir, y después llega el momento en el que hay que salir. De esa posición paciente pasiva se pasa a una posición más activa. Se trata entonces de entender el arte no solamente desde un lugar de entretenimiento (que puede serlo), sino también desde otra arista. Pichon (2007) decía que el artista tiene dos caminos: o afirma una estructura institucional, social o grupal, o 
es un transformador, un contestador, un revolucionario impulsado a crear con el fin de transformar el mundo real, reparando el objeto destruido durante la depresión desencadenada por la enfermedad (Lema, 2014).

En el enfoque dado a este trabajo se integran los contextos que se analizan a partir del método que Guber (2004) define como Etnografía. Este es el caso en la investigación que nos ocupa, y por lo tanto, exige un arduo trabajo de análisis de las propias afectaciones personales, los comportamientos aplicados y las creencias que se movilizan.

Al mismo tiempo, este contacto directo con los escenarios y los actores, a los que Guber llamará "nativos", implica un trabajo de asimilación de sus códigos y perspectivas, a la vez que exige un trabajo de interpretación y descripción científica reservada a la figura del investigador.

El "estar ahi", permite avanzar en la tradicional técnica de la observación, que en sus múltiples versiones ha sido utilizada para explicar la función del investigador, quien, imbuido en el contexto que investiga, observa desde el interior de la escena, permitiéndose el contacto con el campo de una forma absolutamente comprometida y a la vez monitoreada en tanta interferencia.

\section{Metodología}

La presente investigación se enmarca en el paradigma metodológicocualitativo, propio de los estudios exploratorios que intentan conocer en profundidad los fenómenos emergentes. Además, se hace explícito que este recorrido se desarrolla a partir de una perspectiva etnográfica, en su triple registro: desde los escenarios naturales y a partir de la voz propia de los actores, desde los espacios de descripción que estos escenarios y estos actores habilitaron en el investigador, y desde las argumentaciones que fue posible desarrollar para explicar los acontecimientos que se observaron Taylort \& Bogdan, 1986; Vasilachis de Gialdino, 1993). En cuanto al objeto de estudio, Mendicoa, G. (2003) sostiene que su formulación se inicia en el mismo momento en que se define el tema de interés $y$, sobre todo, en el esfuerzo por encontrar las relaciones entre hechos y fenómenos que se presentan en las fuentes empíricas.

\section{Discusión}

La investigación cualitativa permite rescatar la experiencia de la persona, su sentir y su opinión con respecto a lo que se investiga; conocer sus hábitos, costumbres, cultura, maneras de pensar y proceder. Por ello, esta investigación se irá co-construyendo a partir las vivencias cotidianas de los protagonistas. "El mundo social en que vivo como alguien vinculado con otros por múltiples relaciones es para mí un objeto que debe ser interpretado como provisto de sentido" (Alfred, 2012).

La idea central que rige esta premisa ampliamente fundamentada por Sautu (2003), es pensar la investigación como un espacio de producción de conocimiento científico, que abarca mucho más que lo que comúnmente denominamos marco teórico y que implica hacernos cargo de las elecciones argumentales que realizamos y sostenemos para definir y analizar lo que nos ofrece el campo en su despliegue cotidiano.

Luego de la participación en cada taller se realiza el registro en diarios de campo; como ya lo había planteado Malinowski (1972) en su introducción a Los argonautas del Pacífico Occidental, en el registro etnográfico es necesario establecer una clara distinción entre los resultados de la observación directa y las deducciones e interpretaciones del etnógrafo, que estarán basadas en su sentido común, a la vez que atravesadas por el conocimiento científico de la época. Por su parte, Lourau (2004) plantea que ese modo de colecta de datos se constituye a partir de la fusión de tres movimientos que crean tres tipos de diarios diferentes en uno solo: el relevamiento de los datos observados (diario de campo), las impresiones del investigador fruto de sus percepciones e implicaciones (diario íntimo), que finalmente se entrelazan con las especulaciones, proyecciones y construcciones teóricas (el diario de la investigación). Esta herramienta es, en palabras del autor, una "narrativa [...] a omesmo tempo anterior, presente e futuro"(Lourau, 2004), es la producción de un texto científico con la proyección y los esbozos de aquello que está por ser descubierto.

Como se mencionó anteriormente, se privilegia la metodología cualitativa "que tiene en cuenta el contexto en el que actúan los sujetos y la construcción de significados: totalidad, tiempo, lenguaje, interacción, interconexión, ideas propias de dicha metodología"(Sautu, 2003), y como técnicas, la entrevista en profundidad y la observación participante.

\section{En busca de una}

(...) objetividad científica, las posturas positivistas proclaman la neutralidad y el uso aséptico de las técnicas, incurriendo en la manipulación y desdibujamiento de los sujetos de la acción reduciéndolos a datos, respuestas o números. El hablante presente en este tipo de técnicas se asume como un individuo anónimo y como tal puede ser equivalente a otro cualquiera, convirtiéndose en aleatorio e intercambiable" (Tonon, 2005).

"Los datos existen para pensar con ellos y acerca de ellos" 
(Coffey \& Atkinson, 1956), pero son las ideas las que dan la capacidad de aprovechar aquello que se encuentra sin buscarlo.

El Trabajo Social, como disciplina que en sus procesos de intervención se vincula de forma directa con la sociedad y sus problemas, tiene la tarea profesional de trabajar de lleno con problemas de muy diversa índole: necesidades, abusos, violencia, falta de orientación, frustraciones, reclamos y demandas, etcétera, con la convicción de que hay que tener formas más humanistas de entender la realidad, donde no solo nos preocupemos por conocer lo externo de las situaciones y vivencias, sino del sentir del sujeto. Así, se presenta un diseño de investigación desde una perspectiva hermenéutica y etnográfica para describir una realidad, realizando explicaciones particulares del mundo de vida de los sujetos participantes en el estudio de la comunidad, a partir de sus testimonios, los cuales estarán signados por creencias particulares, ubicación histórica, política y relaciones sociales.

La producción de un concepto de Arte, para efectos de esta investigación, requiere de fundamentos teóricos, pues junto con las relaciones sociales, el Arte se vuelve elemento estructural. Escritos sobre el tema encontramos desde los inicios de la historia Occidental, donde personajes como Platón desarrollaban ideas de lo que implica el arte y sus posibles definiciones. Las primeras aproximaciones al concepto de Arte refieren fundamentalmente a la artesanía como oficio, que podía ser ejercido por todo aquel que tuviese destrezas manuales y la capacidad intelectual para este.

Según la bibliografía revisada, en las discusiones del concepto de Arte uno de los elementos que no se ha puesto en duda es que el arte es una actividad humana consciente. De ello da cuenta la conceptualización que hace Tolstoi (1957):

Para dar una definición correcta del arte es necesario cesar de ver en él un material de placer, y considerarle como una de las condiciones de la vida humana. Si se considera así, se advierte que el arte es uno de los medios de comunicación entre los hombres.

En este sentido, el Arte es un proceso humano que implica el desarrollo de la intersubjetividad, entendida esta como la relación sujeto-sujeto, con las condiciones materiales objetivas en las cuales se desarrolla (contexto socioeconómico, históricas). Dentro de esta relación dialéctica de condiciones materiales e inmateriales es que, en determinada posición social (ser y estar en el mundo), el sujeto creador evoca reflexivamente un fragmento de la realidad y, con el proceso artístico creativo, se objetiva un mensaje posible de comunicar a "otro" cierto aspecto de la realidad, a través de símbolos producidos por el sujeto artista, quien objetiva reflexiones, sentidos, pensamientos, ideología, utopía, sentimientos, emociones, etc., en el producto artístico.

El Arte, como proceso creativo, implica una dinamicidad y un cambio permanente, dado el movimiento constante de la realidad social, entendida como el entramado de fuerzas relacionadas contradictoriamente. Es preciso señalar que existe una relación dialéctica entre el Arte y la totalidad de la vida humana, y es que el Arte representa la totalidad de las esferas de la vida, y en ella puede llegar a confluir todo: la ciencia, la tecnología, la educación, los sujetos, etc.

Su riqueza está en la potencialidad que ofrece en términos del mensaje simbólico y la posibilidad de expresión de este en términos estéticos.

\section{Arte como metodología de intervención validad e integral para el ejercicio profesional}

Al momento de ir delimitando el fenómeno de investigación, se logran definir ciertos supuestos, los cuales se comienza a direccionar el presente estudio. Uno de ello, surge con el reconocimiento del Arte, y en particular de las Artes Escénicas, como una posible vía metodológica, a partir de la cual intervenir socialmente. Además, en el Arte se supone la posibilidad de intervenir integralmente con diferentes personas, es decir, una intervención que permitiría relacionar las esferas de lo social, político, económico, cultural e individual.

Como segundo supuesto, se ha definido la posibilidad que existe en el ejercicio artístico de cuestionar el Orden. Este elemento es de vital importancia, dado que, permite relacionarlo con la concepción que se ha ido definiendo de la disciplina profesional del Trabajo Social: la posibilidad transformadora de las relaciones sociales en las que se ven involucradas las personas.

El Arte tiene diferentes conceptualizaciones, matices, perspectivas, miradas, pero desde esta investigación nos interesa el Arte desde su dimensión social. Partiendo desde este punto, se construirá la conceptualización sobre esta categoría, para constatar si es pertinente como espacio de intervención para nuestra profesión.

Si el Arte está en el aire, si se siente, se vive, se ve; es parte de plazas, barrios, comunidades, ciudades, también dentro de las instituciones (como hospitales, penitenciarias, centros de salud, municipios, escuelas, centros culturales, uniones vecinales). Si es algo que se está dando, que fluye, ¿por qué no considerarlo desde el Trabajo Social como un espacio pertinente de intervención? ¿Por qué no 
pensarlo como un espacio que posibilite la construcción de soluciones alternativas, con el fin de movilizar y transformar la situación problemática que plantean los colectivos con los cuales se trabaja?

Al ingresar al hospital, el sujeto es separado de su mundo habitual, es despojado de sus posesiones materiales (las pertenencias de valor por cuestiones de seguridad son entregadas a los familiares y tanto el Documento Nacional de Identidad como cualquier otra documentación relevante se guarda en el office de los profesionales), sólo les pertenece lo que llevan puesto; es en este proceso donde también es despojado de los roles y vínculos que hasta entonces daban sentido a su existencia cotidiana.

Un conjunto de pertenencias de un individuo tiene especial relación con su yo. El individuo espera generalmente controlar de algún modo el aspecto que presenta ante los demás. Para esto necesita varios artículos de tocador, y varias mudas de ropa, elementos para adaptarlas, disponerlas y repararlas, y un lugar accesible y seguro donde guardar estas reservas. En síntesis, el individuo necesitará un equipo de identificación que guiará el manejo de su apariencia personal. Al ingresar en una institución, probablemente se le despoje de su acostumbrada apariencia, así como de los instrumentos y servicios con los que la mantiene, esto posibilita al individuo que sufra una desfiguración personal (Goffman, 2001).

El paisaje del hospital sacude arrebatando el aire: muros, paredes con humedad, colchones rotos, ropa sucia, ventanas rotas, invisibilidad latente, y una rutina que no da lugar al acontecer de lo espontaneo, de la sorpresa. Cuando la continuidad de la vida es rechazada, o no necesita ser rechazada porque existe de antemano una extrañeza, una alteridad, un estar al margen, es cuando se habla de aventura (Simmel, 1988). El arte es una aventura, una instancia de ruptura en la cotidianeidad asfixiante de la institución. Una experiencia que se desprende del proceso homogéneo de la vida cotidiana y que, si bien se vincula directamente hacia adelante y hacia atrás con otras experiencias, en su sentido más profundo y quizás más doloroso discurre al margen de la continuidad propia de la vida en el hospital. Hay un espacio-tiempo en el que el sujeto puede hacer y lo que es más significativo, ser a partir del hacer (no es casual que el arte sea el lugar al que quieran asistir apenas traspasan las puertas del psiquiátrico).

Al hacer circular las producciones artísticas (materiales y no) se producen efectos subjetivos, institucionales $y$ sociales. A personas que el manicomio les ha socavado sus deseos, sus pasiones y sus proyectos de vida, el arte las convoca a un trabajo grupal dentro de un proceso creador.
Lo que circula no es solo la producción, sino el artista. De esta manera, la persona puede decir lo que le pasa dentro del hospital, mencionar lo que funciona y denunciar los aspectos negativos y los malos tratos físicos y psíquicos, la sobremedicación y la falta de libertad. Estas denuncias muchas veces vuelven al hospital y es allí en donde se produce un efecto institucional. En principio, poniendo en evidencia las contradicciones institucionales y abriendo dentro del hospital nuevas grietas que permitan profundizar el debate sobre estas problemáticas. Es esta contradicción la que interpela nuestras intervenciones profesionales cotidianas.

Así, ¿el arte es medio o fin en sí mismo? ¿Resulta necesario elegir temáticas para que sean trabajadas a través del arte plástico por el trabajador social o simplemente el hecho de proponer "hacer arte" trae aparejada implícitamente las estrategias de intervención profesional?

\section{Conclusiones}

La propuesta de innovar a través del arte no resulta solo una invitación de corte metodológico, sino que incita a renovar algunas aseveraciones históricas del Trabajo Social como la necesidad de "hacer", la posición de la visita domiciliaria como técnica representativa del colectivo profesional, y la vinculación de instituciones como espacio de inserción al asistencialismo.

El increíble protagonismo que asume y se le adjudica al arte plástico en las intervenciones de las que forma parte, a través de modalidades como la pintura individual y la mural, es aquello que produce la impactante influencia en las demás aristas de la actuación profesional misma: la población, el espacio físico, el marco institucional, los recursos, el perfil profesional y hasta las temáticas de trabajo que asumen un papel casi inadvertido, avasalladas por la reflexión que el mismo arte plástico trae aparejada.

Estos aportes recapitulados son los que permiten que aparezca la necesidad de asociar al arte plástico como herramienta del Trabajo Social con el disfrute y con el ocio, especialmente frente a poblaciones en contextos en crisis, o de padecimiento subjetivo, donde la necesidad se trasforma en urgencia, donde el uso del tiempo libre, abundante en estas circunstancias, corre el riesgo de devenir en desgaste, desuso y hasta deformación.

Más allá de funcionar como herramienta promotora de la creatividad y del disfrute, el arte plástico puede operar como mecanismo preventivo no solo del mencionado desgaste profesional sino también de las posibles repercusiones que este genera en las poblaciones con las que trabajamos. 
El objetivo propuesto es ambicioso, es también audaz, y, si se quiere, insolente. Se enfrenta a intervenciones instaladas, cuestiona estrategias, se permite la disidencia, la creación, la construcción a partir de la deconstrucción, se permite desandar, inventar y reinventar, tal vez, para no dejarse atrapar.

\section{Referencias}

Alberdi, J. (2012) Desafíos políticos y asistenciales de la nueva ley nacional de salud mental. El caso de la Provincia de Santa. Revista Debate Público. Reflexión de Trabajo Socia, 2(4), 93-105.

Alfred, S. (2012). Estudios sobre teoría social. Escritos II. Buenos Aires: Amorrortu.

Amarante, P., Freitas, F., Pande, M. R., \& Nabuco, E. (2013). El campo artístico-cultural en la reforma psiquiátrica brasileña: el paradigma identitario del reconocimiento. Salud Colectiva, 9, 287-299.

Amico, L. D. (2005). La institucionalización de la locura. (Espacio Editorial, Ed.). Buenos Aires.

Barg, L. (2006). Construyendo la política institucional de un Centro de Salud Mental Infanto Juvenil. In Espacio Editorial (Ed.), Lo interdisciplinario en salud mental: niños, adolescentes, sus familias y la comunidad (pp. 15-28).

Birman, J., \& Serra, A. (1988). Exclusão social do doente mental: discursos e representações no contexto da reforma psiquiátrica Silvana Carneiro Maciel. Universidad Federal da Paraíba.

Boal, A. (2004). El arco iris del deseo: del teatro experimental a la terapia. España: Alba editorial.

Carballeda, A. (2007). La intervención en lo social. Exclusión e intervención en los nuevos escenarios sociales. Buenos Aires: Paidós.

Carballeda, A. (2008). Los cuerpos fragmentados. La intervención en lo social y en los escenarios de la exclusión y el desencanto. Buenos Aires: Paidós.

Cazzaniga, S. (2006). Intervención profesional: legitimidades en debate. Buenos Aires: Espacio Editorial.

Clark, L. (1998). L'art c'est le corps. Lygia Clark. Barcelona: Fundació Antoni Tàpies e Edition de L'eixample.

Coffey, A., \& Atkinson, P. (1956). Encontrar el sentido a los datos cualitativos. Estrategias complementarias de investigación, Colombia. Universidad de Antioquia.

Deleuze, G. (1996). Crítica y clínica. Barcelona: Anagrama.

Deleuze, G., \& Bacon, F. (2002). Lógica de la sensación. Madrid: Editora Nacional.

Forni, F., Gallart, M., \& Vasilachis de Gialdino, I. (1993). Estrategias de recolección y estrategias de análisis en la investigación social. Buenos Aires: Centro Editor de América Latina.

Foucault, M. (1961). Maladie Mentale Et Personnalite. Paris: PUF.

Foucault, M. (1998). Historia de la locura en la Época Clásica. México: FCE.
Galende, E. (1997). De un horizonte incierto. Psicoanálisis y Salud Mental en la sociedad actual. Buenos Aires: Paidós.

Goffman, E. (1961). Asylums: essays on the soocial situatioon of mental patients and other inmates.

Goffman, E. (1974). Análisis del marco: un ensayo sobre la organización de la experiencia. Harvard University Press.

Goffman, E. (1997). Presentación de la persona en la vida cotidiana. Buenos Aires: Amorrortu.

Goffman, E. (2001). Internados: Ensayos sobre la situación social de los enfermos mentales. Buenos Aires: Amorrourtu.

Gónzalez Saibene, A. (2007). Acerca de la intervención y otros textos. Documento Jornadas Docentes sobre la intervención, Escuela de Trabajo Social. Facultad Cs Pol y RRII.UNR.

Grotowski, J. (1992). Hacia un teatro pobre. Buenos Aires: Siglo Veintiuno.

Guattari, F., \& Rolink, S. (2006). Micropolitica. Cartografías del deseo. Madrid: Traficantes de sueños.

Guber, R. (2004). El salvaje metropolitano: Reconstrucción del conocimiento social en el trabajo de campo. Buenos Aires: Paidós.

Kandinski, W. (1989). De lo espiritual en el arte. México: Premia. Kisnerman, N. (1984). Comunidad. Buenos Aires: Humanitas. Kraepelin, E. (2008). La demencia precoz. Buenos Aires: Polemos.

Lema, V. (2014). Conversaciones con Enrique Pichon Riviere sobre el arte y la locura. Buenos Aires: Ediciones Cinco.

Lourau, R. (2004). Analista institucional em tempo integral. San Pablo: Hucitec.

Malinowski, B. (1972). Los argonautas del pacifico occidental. Barcelona: Planeta Agostini.

Marradi, A., Archenti, N., \& Piovani, J. I. (2007). Metodología de las ciencias sociales. Buenos Aires.

Mendicoa, G. (2003). Sobre Tesis y tesistas, Lecciones de Enseñanza Aprendizaje. Buenos Aires: Espacio.

Mengue, P. (2008). People and fabulation. Deleuze and politics. Ministerio de Salud. (2013). Ley Nacional de salud mental No. 26.657. Recuperado de http://www.minsa.gob.pa/sites/ default/files/programas/guia_medica_de_atencion_ integra_am_digital.pdf

Murekian, N. (2002). Salud mental, fuentes de información y representaciones sociales: el peso de los factores estructurales en el anclaje diferencial del impacto cognoscitivo y simbólico de las políticas de desmanicomializacion. Recuperado de http://catalogosuba.sisbi.uba.ar/vufind/ Record/201603170441373632

Pichon Rivière, E. (2007). El proceso grupal. Del psicoanálisis a la psicología social. Editorial Nueva Visión.

Richmond, M. (1917). The social case worker's task. In Proceedings of the National Conference of Social Work. Chicago: Rogers \& Hall Co.

Rozas Pagaza, M. (2001). La intervención profesional en relación con la cuestión social. Buenos Aires: Espacio. 
Sautu, R. (2003). Todo es teoría. Objetivos y métodos de investigación. Buenos Aires: Lumiere.

Simmel, G. (1988). Sobre la aventura ensayos filosóficos. Barcelona.

Taylort, J., \& Bogdan, R. (1986). Introducción a los métodos cualitativos de investigación. La búsqueda de significados. Buenos Aires: Paidós.

Tolstoi, L. (1957). Qué es el arte. Tor.

Tonon, G. (2005). Las técnicas de actuación profesional del Trabajo Social. Buenos Aires: Espacio.

UNESCO. (2016). Antonio Berni. Recuperado de https:// redbioetica.com.ar/antonio-berni/

Vasilachis de Gialdino, I. (1993). Métodos Cualitativos I. Los problemas teórico epistemológicos. Buenos Aires: Centro Editor de América Latina.

Velez Restrepo, O. (2003). Reconfigurando el Trabajo Social. Perspectivas y tendencias contemporáneas. Buenos Aires: Espacio. 